EFI-91-57

\title{
Some Exact Solutions of String Theory in Four and Five Dimensions
}

\author{
Petr Hořava* \\ Enrico Fermi Institute \\ University of Chicago \\ 5640 South Ellis Avenue \\ Chicago, IL 60637, USA \\ e-mail: horava@yukawa.uchicago.edu
}

\begin{abstract}
We find several classes of exact classical solutions of critical bosonic string theory, constructed as twisted products of one Euclidean and one Minkowskian 2D black hole coset. One class of these solutions leads (after tensoring with free scalars and supersymmetrizing) to a rotating version of the recently discovered exact black fivebrane. Another class represents a one-parameter family of axisymmetric stationary four-dimensional targets with horizons. Global properties and target duality of the 4D solutions are briefly analyzed.
\end{abstract}

October 1991

* Robert R. McCormick Fellow; work also supported by the NSF under Grant No. PHY9000386; the DOE under Grant No. DEFG02-90ER40560; the Czechoslovak Chart 77 Foundation; and the ČSAV under Grant No. 91-11045. 


\section{Introduction}

Exact solutions of (perturbative) string theory that do not represent strings in static spacetimes with flat time coordinate (plus possible internal degrees of freedom), but rather describe spacetimes of nontrivial metric properties, have attracted much interest recently. One of the most excellent examples are Witten's black holes in 2D string theory [1], as well as their generalizations to charged black holes in 2D [2], black strings in 3D [3], and exact fivebranes in 10D superstring theory [4]. With these solutions at hand, one naturally wonders whether the techniques can be used to construct exact solutions of string theory in four dimensions.

To answer this question in the affirmative, let us start with Witten's black hole coset in 2D spacetime with Minkowskian signature. We might obtain a model which describes strings in a 4D manifold with Minkowskian signature by tensoring the 2D black hole with another conformal field theory, describing strings in a 2D manifold of Euclidean signature. Obviously, we have one excellent candidate for such a manifold: The 2D black hole itself, now in the Euclidean regime.

To get a nontrivial 4D spacetime, we would like to allow a 'twist' in the product of the two conformal field theories. Technically, the basic idea of this paper is to start with a direct product of two WZW models, and gauge a group that acts nontrivially on both of them, thus producing a conformal field theory that is no longer a direct product (compare [3]). In the case of two 2D black holes, a naive way of doing so might be as follows. Starting with the direct product of two $S L(2, \mathbf{R})$ WZW models (referred to as $S L(2, \mathbf{R})_{M, E}$ throughout),

$$
\begin{aligned}
\mathcal{L}_{\mathrm{WZW}}= & \frac{i k_{M}}{4 \pi} \int d^{2} z \operatorname{Tr}\left(g_{M}^{-1} \partial g_{M} g_{M}^{-1} \bar{\partial} g_{M}\right)-i k_{M} \Gamma\left(g_{M}\right) \\
& +\frac{i k_{E}}{4 \pi} \int d^{2} z \operatorname{Tr}\left(g_{E}^{-1} \partial g_{E} g_{E}^{-1} \bar{\partial} g_{E}\right)-i k_{E} \Gamma\left(g_{E}\right)
\end{aligned}
$$

where $g_{M, E} \in S L(2, \mathbf{R})_{M, E}$, we will gauge two Abelian symmetry groups of the model. First, we will gauge the compact Abelian group

$$
g_{E} \rightarrow h_{E} g_{E} h_{E}
$$

with $h_{E}$ generated by $\left(\begin{array}{cc}0 & 1 \\ -1 & 0\end{array}\right)$. At this stage, we get a direct product of the $2 \mathrm{D}$ black hole coset with Euclidean signature in sector $E$ and the ungauged WZW model in sector $M$. In the second step, we will gauge the noncompact group

$$
g_{M} \rightarrow h_{M} g_{M} h_{M}, \quad g_{E} \rightarrow h_{E}^{\alpha} g_{E} h_{E}^{-\alpha}
$$


with $h_{M}$ generated by $\left(\begin{array}{cc}1 & 0 \\ 0 & -1\end{array}\right)$. Here $\alpha \in \mathbf{R}$ is a 'distortion parameter': For $\alpha=0$, we get a direct product of one Euclidean and one Minkowskian 2D black hole.

The total conformal anomaly of the gauged model is

$$
c=\frac{3 k_{M}}{k_{M}-2}+\frac{3 k_{E}}{k_{E}-2}-2 .
$$

To get a critical theory in four dimensions, we set $c=26$. This condition restricts the values of $k_{M, E}$ to

$$
k_{M, E}=k \pm \tilde{k}
$$

with

$$
\tilde{k}= \pm \sqrt{\left(k-\frac{28}{11}\right)(k-2)} .
$$

We will further restrict ourselves to

$$
k \geq \frac{28}{11}
$$

to avoid complex values of the levels.1

Let us parametrize the group manifolds by their Euler angles:

$$
\begin{aligned}
g_{M} & =\left(\begin{array}{cc}
e^{t_{L} / 2} & 0 \\
0 & e^{-t_{L} / 2}
\end{array}\right)\left(\begin{array}{ll}
\cosh \frac{r_{M}}{2} & \sinh \frac{r_{M}}{2} \\
\sinh \frac{r_{M}}{2} & \cosh \frac{r_{M}}{2}
\end{array}\right)\left(\begin{array}{cc}
e^{-t_{R} / 2} & 0 \\
0 & e^{t_{R} / 2}
\end{array}\right), \\
g_{E} & =\left(\begin{array}{cc}
\cos \frac{\theta_{L}}{2} & \sin \frac{\theta_{L}}{2} \\
-\sin \frac{\theta_{L}}{2} & \cos \frac{\theta_{L}}{2}
\end{array}\right)\left(\begin{array}{ll}
\cosh \frac{r_{E}}{2} & \sinh \frac{r_{E}}{2} \\
\sinh \frac{r_{E}}{2} & \cosh \frac{r_{E}}{2}
\end{array}\right)\left(\begin{array}{cc}
\cos \frac{\theta_{R}}{2} & -\sin \frac{\theta_{R}}{2} \\
\sin \frac{\theta_{R}}{2} & \cos \frac{\theta_{R}}{2}
\end{array}\right),
\end{aligned}
$$

with $r_{M} \in \mathbf{R}, r_{E} \in[0, \infty), t_{L, R} \in \mathbf{R}$, and $\theta_{L, R} \in[0,2 \pi]$, 2 and denote the gauge fields that correspond to (1.2) and (1.3) by $A_{E}, \bar{A}_{E}$ and $A_{M}, \bar{A}_{M}$ respectively. Upon choosing a unitary gauge by setting

$$
t_{L}=t_{R} \equiv t, \quad \theta_{L}=\theta_{R} \equiv \theta
$$

1 We have omitted here the other region with real $k_{M, E}$, namely $k \leq \frac{8}{5}$. This region would correspond to the analytic continuation of one of the $S L(2, \mathbf{R})$ 's to $S U(2)$ (see below).

2 Two facts seem worth stressing. First, the Euler angle parametrization of $g_{M}$ we have used does not cover the whole $S L(2, \mathbf{R})_{M}$ manifold. Second, the ranges for $\theta_{L, R}, r_{E}$ cover $S O(2,1)$ rather than its double cover $S L(2, \mathbf{R})$. 
we arrive at the following Lagrangian:

$$
\begin{aligned}
\mathcal{L}_{4 \mathrm{D}}= & \mathcal{L}_{\mathrm{WZW}}+\frac{i k_{M}}{\pi} \int d^{2} z \sinh ^{2} \frac{r_{M}}{2}\left(\bar{A}_{M} \partial t-A_{M} \bar{\partial} t\right) \\
& +\frac{2 i k_{M}}{\pi} \int d^{2} z \cosh ^{2} \frac{r_{M}}{2} A_{M} \bar{A}_{M} \\
& +\frac{i k_{E}}{\pi} \int d^{2} z \sinh ^{2} \frac{r_{E}}{2}\left(A_{E} \bar{\partial} \theta-\bar{A}_{E} \partial \theta+\alpha A_{M} \bar{\partial} \theta+\alpha \bar{A}_{M} \partial \theta\right) \\
& +\frac{2 i k_{E}}{\pi} \int d^{2} z\left(\alpha^{2} \sinh ^{2} \frac{r_{E}}{2} A_{M} \bar{A}_{M}-\cosh ^{2} \frac{r_{E}}{2} A_{E} \bar{A}_{E}\right) \\
& +\frac{i k_{E}}{\pi} \int d^{2} z \alpha\left(A_{E} \bar{A}_{M}-A_{M} \bar{A}_{E}\right)\left(\cosh ^{2} \frac{r_{E}}{2}+\sinh ^{2} \frac{r_{E}}{2}\right)
\end{aligned}
$$

As the gauge fields enter quadratically, they can be integrated out by solving their equations of motion. The final (lowest order) Lagrangian reads

$$
\begin{aligned}
\mathcal{L}_{4 \mathrm{D}}=\frac{i}{2 \pi} \int d^{2} z & \left(\frac{k_{E}}{4} \partial r_{E} \bar{\partial} r_{E}+\frac{k_{E} \sinh ^{2} \frac{r_{M}}{2}\left(\cosh ^{2} \frac{r_{E}}{2}-K\right)}{\cosh ^{2} \frac{r_{M}}{2} \cosh ^{2} \frac{r_{E}}{2}-K} \partial \theta \bar{\partial} \theta\right. \\
& +\frac{k_{M}}{4} \partial r_{M} \bar{\partial} r_{M}-k_{M} \frac{\sinh ^{2} \frac{r_{E}}{2}\left(\cosh ^{2} \frac{r_{M}}{2}-K\right)}{\cosh ^{2} \frac{r_{M}}{2} \cosh ^{2} \frac{r_{E}}{2}-K} \partial t \bar{\partial} t \\
& \left.+\frac{\alpha k_{E} \sinh ^{2} \frac{r_{M}}{2} \sinh ^{2} \frac{r_{E}}{2}}{2\left(\cosh ^{2} \frac{r_{M}}{2} \cosh ^{2} \frac{r_{E}}{2}-K\right)}(\partial \theta \bar{\partial} t-\bar{\partial} \theta \partial t)\right)
\end{aligned}
$$

with $K$ a shorthand for $4 \alpha^{2} k_{E} / k_{M}$. Obviously, it describes strings in a $4 \mathrm{D}$ target, with the following metric and antisymmetric tensor background:

$$
\begin{aligned}
d s^{2}=\frac{k_{E}}{4} d r_{E}^{2}+\frac{k_{M}}{4} d r_{M}^{2} & +k_{E} \frac{\sinh ^{2} \frac{r_{M}}{2}\left(\cosh ^{2} \frac{r_{E}}{2}-K\right)}{\cosh ^{2} \frac{r_{M}}{2} \cosh ^{2} \frac{r_{E}}{2}-K} d \theta^{2} \\
& -k_{M} \frac{\sinh ^{2} \frac{r_{E}}{2}\left(\cosh ^{2} \frac{r_{M}}{2}-K\right)}{\cosh ^{2} \frac{r_{M}}{2} \cosh ^{2} \frac{r_{E}}{2}-K} d t^{2} \\
B= & \frac{\alpha k_{E} \sinh ^{2} \frac{r_{M}}{2} \sinh ^{2} \frac{r_{E}}{2}}{2\left(\cosh ^{2} \frac{r_{M}}{2} \cosh ^{2} \frac{r_{E}}{2}-K\right)} d \theta \wedge d t
\end{aligned}
$$

The nontrivial determinant coming from the integration over the gauge fields leads to a dilaton background,

$$
\Phi=\ln \left(\cosh ^{2} \frac{r_{M}}{2} \cosh ^{2} \frac{r_{E}}{2}-K\right)+\text { const }
$$

Throughout the paper, we will only consider sigma-model metrics. The so-called canonical metrics can be obtained from the sigma-model metrics by a proper rescaling by an exponential of $\Phi$. 
A priori we might have expected that the $4 \mathrm{D}$ theory would have an exact $U(1) \times U(1)$ symmetry. Indeed, two abelian symmetries survive our gauging of the WZW model. Without any additional arguments, our conformal field theory seems to represent a class of exact stationary and axisymmetric solutions of string theory in four dimensions. Unfortunately, this is not true, the reason being that the gauging we have attempted to do is in fact anomalous. Indeed [5], we can easily check that the anomaly-cancellation condition,

$$
\operatorname{Tr}\left(T_{a, L} T_{b, L}-T_{a, R} T_{b, R}\right)=0
$$

is not met. (Here we have used the notation of [5], i.e. $a, b$ are gauge group indices and $T_{a, L}, T_{a, R}$ generate the gauge group action on the WZW fields, $\delta g=\epsilon^{a}\left\{T_{a, L} \cdot g+g\right.$. $\left.T_{a, R}\right\}$.) We thus cannot hope that (1.12) is more than a solution of the low-energy effective approximation to string theory.

To obtain genuine solutions of string theory, we will modify our basic strategy in two directions. First, we will obtain in section 2 a class of models in five dimensions, simply by gauging the $U(1)$ group that acts on both of the $S L(2, \mathbf{R})$ 's, and forgetting about the other $U(1)$. As we will see, this leads (after continuing analytically, tensoring with free bosons, and supersymmetrizing) to rotating versions of the recently discovered [6] [4]

fivebrane solitons of superstring theory. Second, in section 3 we will modify the action of $U(1) \times U(1)$ so as to avoid the violation of Witten's condition of non-anomalousness. This will result in a class of exact, stationary and axisymmetric solutions in four dimensions.

\section{Exact Rotating Black Fivebranes from 2D Black Holes}

Let us now start with the tensor product Lagrangian (1.1), and gauge the $U(1)$ group acting by

$$
g_{E} \rightarrow h_{E} g_{E} h_{E}, \quad g_{M} \rightarrow h_{M}^{\beta} g_{M} h_{M}^{\beta}
$$


with $\beta$ a distortion parameter. Upon gauging this group, the Lagrangian becomes

$$
\begin{aligned}
\mathcal{L}= & \mathcal{L}_{\mathrm{WZW}}+\frac{i k_{E}}{2 \pi} \int d^{2} z A \operatorname{Tr}\left(\left(\begin{array}{cc}
0 & 1 \\
-1 & 0
\end{array}\right) \bar{\partial} g_{E} g_{E}^{-1}\right) \\
& +\frac{i k_{E}}{2 \pi} \int d^{2} z \bar{A} \operatorname{Tr}\left(\left(\begin{array}{cc}
0 & 1 \\
-1 & 0
\end{array}\right) g_{E}^{-1} \partial g_{E}\right) \\
& +\frac{i k_{E}}{2 \pi} \int d^{2} z A \bar{A}\left[-2+\operatorname{Tr}\left(\left(\begin{array}{cc}
0 & 1 \\
-1 & 0
\end{array}\right) g_{E}\left(\begin{array}{cc}
0 & 1 \\
-1 & 0
\end{array}\right) g_{E}^{-1}\right)\right] \\
& +\frac{i k_{M}}{2 \pi} \int d^{2} z \beta A \operatorname{Tr}\left(\left(\begin{array}{cc}
1 & 0 \\
0 & -1
\end{array}\right) \bar{\partial} g_{M} g_{M}^{-1}\right) \\
& +\frac{i k_{M}}{2 \pi} \int d^{2} z \beta \bar{A} \operatorname{Tr}\left(\left(\begin{array}{cc}
1 & 0 \\
0 & -1
\end{array}\right) g_{M}^{-1} \partial g_{M}\right) \\
& +\frac{i k_{M}}{2 \pi} \int d^{2} z \beta^{2} A \bar{A}\left[2+\operatorname{Tr}\left(\left(\begin{array}{cc}
1 & 0 \\
0 & -1
\end{array}\right) g_{M}\left(\begin{array}{cc}
1 & 0 \\
0 & -1
\end{array}\right) g_{M}^{-1}\right)\right]
\end{aligned}
$$

where we have denoted by $A, \bar{A}$ the gauge field associated with (2.1). Upon parametrizing the group manifolds as in (1.8) and fixing the gauge by

$$
\theta_{L}=\theta_{R} \equiv \theta
$$

we observe that the model describes the following five-dimensional background (to lowest order):

$$
\begin{aligned}
d s_{5 \mathrm{D}}^{2}= & \frac{k_{M}}{4} d r_{M}^{2}+\frac{k_{E}}{4} d r_{E}^{2}+\frac{k_{E} \sinh ^{2} \frac{r_{E}}{2}\left(1-L \cosh ^{2} \frac{r_{M}}{2}\right)}{\cosh ^{2} \frac{r_{E}}{2}-L \cosh ^{2} \frac{r_{M}}{2}} d \theta^{2} \\
& +\frac{k_{M} \sinh ^{2} \frac{r_{M}}{2}\left(L-\cosh ^{2} \frac{r_{E}}{2}\right)}{\cosh ^{2} \frac{r_{E}}{2}-L \cosh ^{2} \frac{r_{M}}{2}} d t^{2}+\frac{2 k_{M} \beta \sinh ^{2} \frac{r_{E}}{2} \sinh ^{2} \frac{r_{M}}{2}}{\cosh ^{2} \frac{r_{E}}{2}-L \cosh ^{2} \frac{r_{M}}{2}} d t d \theta \\
& \quad+\frac{k_{M} \cosh ^{2} \frac{r_{M}}{2} \cosh ^{2} \frac{r_{E}}{2}}{\cosh ^{2} \frac{r_{E}}{2}-L \cosh ^{2} \frac{r_{M}}{2}} d \tilde{t}^{2} \\
B= & \frac{k_{M} \sinh ^{2} \frac{r_{M}}{2} \cosh ^{2} \frac{r_{E}}{2}}{\cosh ^{2} \frac{r_{E}}{2}-L \cosh ^{2} \frac{r_{M}}{2}} d t \wedge \tilde{t}-\frac{\beta k_{M} \sinh ^{2} \frac{r_{E}}{2} \cosh ^{2} \frac{r_{M}}{2}}{\cosh ^{2} \frac{r_{E}}{2}-L \cosh ^{2} \frac{r_{M}}{2}} d \theta \wedge d \tilde{t}, \\
\Phi= & \ln \left(\cosh ^{2} \frac{r_{E}}{2}-L \cosh ^{2} \frac{r_{M}}{2}\right)+\operatorname{const}
\end{aligned}
$$

where $t, \tilde{t}=\frac{1}{2}\left(t_{L} \pm t_{R}\right)$ and $L=\beta^{2} k_{M} / k_{E}$. As (2.3) does not fix the gauge completely, $\tilde{t}$ is orbifoldized, and $\tilde{t} \equiv \tilde{t}+2 \pi \beta$.

While this geometry is interesting in itself, we can find connections to some results obtained recently [6], [4], [7]- [1]] by continuing it analytically to a gauged $S L(2, \mathbf{R}) \times S U(2)$ WZW model. Upon parametrizing the $S U(2)$ group manifold by its Euler angles,

$$
g=\left(\begin{array}{cc}
e^{i \theta_{L} / 2} & 0 \\
0 & e^{-i \theta_{L} / 2}
\end{array}\right)\left(\begin{array}{cc}
\cos \frac{\phi}{2} & i \sin \frac{\phi}{2} \\
i \sin \frac{\phi}{2} & \cos \frac{\phi}{2}
\end{array}\right)\left(\begin{array}{cc}
e^{-i \theta_{R} / 2} & 0 \\
0 & e^{i \theta_{R} / 2}
\end{array}\right)
$$


with $\phi \in[0, \pi)$ and the ranges for $\theta_{L, R}$ as before, we can see that the corresponding gauged model is related to the one constructed previously, by the analytic continuation of $r_{E}$ to $\phi$ via $r_{E}=i \phi$. In addition, this analytic continuation has to be supplemented with the sign reversal of the level, in order to preserve the relative metric signature of the two group manifolds. We will thus assume $k \equiv-k_{E} \geq 0$ henceforth, as well as reverse the sign of $L$ so as to ensure $L \geq 0$. The central charge of the model is

$$
c=\frac{3 k_{M}}{k_{M}-2}+\frac{3 k}{k+2}-1
$$

and $k$ is restricted by unitarity to a discrete set of values, as usual. (We don't set $c=26$ here, as it is more interesting to tensor the model with five free scalars and supersymmetrize it. Imposing $c_{\text {tot }}=15$ afterwards, we obtain a solution of $N=1$ superstring theory.) With these conventions, we get

$$
\begin{aligned}
& d s_{5 \mathrm{D}}^{2}= \frac{k_{M}}{4} d r_{M}^{2}+\frac{k}{4} d \phi^{2}+\frac{k \sin ^{2} \frac{\phi}{2}\left(1+L \cosh ^{2} \frac{r_{M}}{2}\right)}{\cos ^{2} \frac{\phi}{2}+L \cosh ^{2} \frac{r_{M}}{2}} d \theta^{2} \\
&-\frac{k_{M} \sinh ^{2} \frac{r_{M}}{2}\left(L+\cos ^{2} \frac{\phi}{2}\right)}{\cos ^{2} \frac{\phi}{2}+L \cosh ^{2} \frac{r_{M}}{2}} d t^{2}-\frac{2 k_{M} \beta \sin ^{2} \frac{\phi}{2} \sinh ^{2} \frac{r_{M}}{2}}{\cos ^{2} \frac{\phi}{2}+L \cosh ^{2} \frac{r_{M}}{2}} d t d \theta \\
& \quad+\frac{k_{M} \cosh ^{2} \frac{r_{M}}{2} \cos ^{2} \frac{\phi}{2}}{\cos ^{2} \frac{\phi}{2}+L \cosh ^{2} \frac{r_{M}}{2}} d \tilde{t}^{2} \\
& B=\frac{k_{M} \sinh ^{2} \frac{r_{M}}{2} \cos ^{2} \frac{\phi}{2}}{\cos ^{2} \frac{\phi}{2}+L \cosh ^{2} \frac{r_{M}}{2}} d t \wedge d \tilde{t}+\frac{\beta k_{M} \sin ^{2} \frac{\phi}{2} \cosh ^{2} \frac{r_{M}}{2}}{\cos ^{2} \frac{\phi}{2}+L \cosh ^{2} \frac{r_{M}}{2}} d \theta \wedge d \tilde{t} \\
& \Phi=\ln \left(\cos ^{2} \frac{\phi}{2}+L \cosh ^{2} \frac{r_{M}}{2}\right)+\text { const. }
\end{aligned}
$$

This model is a rotating analog of the fivebrane soliton discovered in 6 四. The core of the fivebrane is surrounded by a spherical horizon 3 localized at $r_{M}=0$. At fixed $t$, the horizon inherits the following metric:

$$
d s_{\text {horizon }}^{2}=\frac{k}{4} d \phi^{2}+\frac{k \sin ^{2} \frac{\phi}{2}(1+L)}{\cos ^{2} \frac{\phi}{2}+L} d \theta^{2}+\frac{k_{M} \cos ^{2} \frac{\phi}{2}}{\cos ^{2} \frac{\phi}{2}+L} d \tilde{t}^{2} .
$$

The exact black fivebrane of [4] has the structure of the direct product of $S U(2)$ and $S L(2, \mathbf{R}) / U(1)$, which we recover in the limit of $L \rightarrow \infty$.

3 Our coordinates $\phi, \theta, \tilde{t}$ do parametrize a 3-sphere, albeit in an unusual manner. More standard coordinates on the sphere would result from an alternative gauge choice in the gauged WZW model, namely $t_{L}=t_{R} \equiv 0$. 
It is worth noting that the model is indeed not asymptotically flat, rather it is asymptotic to $S^{3} \times \mathbf{R}$, as can be seen in the $r_{M} \rightarrow \infty$ limit of the metric: $\mathbb{A}^{-}$

$$
\begin{aligned}
d s_{5 \mathrm{D}}^{2} \rightarrow \frac{k_{M}}{4} d r_{M}^{2} & +\frac{k}{4} d \phi^{2}+k \sin ^{2} \frac{\phi}{2} d \theta^{2}-\left(k_{M}+\frac{k}{\beta^{2}} \cos ^{2} \frac{\phi}{2}\right) d t^{2} \\
& -\frac{2 k}{\beta} \sin ^{2} \frac{\phi}{2} d t d \theta+\frac{k}{\beta^{2}} \cos ^{2} \frac{\phi}{2} d \tilde{t}^{2}
\end{aligned}
$$

We can also observe dragging of inertial frames, a typical effect of rotating bodies in general relativity.

As we have argued that our class of conformal field theories represents essentially a rotating deformation of the fivebrane solution constructed by Giddings and Strominger, it is natural to look for the exact marginal vertex operator that governs this deformation. In the approximation of (2.7), the vertex operator can be easily identified as

$$
V_{\operatorname{marg}} \sim \frac{i k}{2 \pi} \int d^{2} z \sin ^{2} \frac{\phi}{2}\left[\tanh ^{2} \frac{r_{M}}{2}(\partial t \bar{\partial} \theta+\bar{\partial} t \partial \theta)-(\partial \theta \bar{\partial} \tilde{t}-\bar{\partial} \theta \partial \tilde{t})\right]
$$

This indeed represents a lowest order approximation to an exactly margninal operator. The exact form of the operator can be identified by looking at the full-fledged coset Lagrangian, leading to

$$
V_{\operatorname{marg}}=\frac{i k}{2 \pi} \int d^{2} z\left[A \operatorname{Tr}\left(\left(\begin{array}{cc}
0 & 1 \\
-1 & 0
\end{array}\right) \bar{\partial} g_{E} g_{E}^{-1}\right)+\bar{A} \operatorname{Tr}\left(\left(\begin{array}{cc}
0 & 1 \\
-1 & 0
\end{array}\right) g_{E}^{-1} \partial g_{E}\right)\right]
$$

One can easily check that after integrating out the gauge field in (2.11), one arrives at (2.10). Note the interesting fact that the exactly marginal vertex operator (2.11) acts on the conformal field theory of the non-rotating fivebrane (which corresponds to the limit of $L \rightarrow \infty$ in our parametrization) by redefining the BRST charge, thus leading to a one-parametric class of deformations of the BRST cohomology of the model.

Recalling that $\phi \in[0, \pi)$, we can see that the set of coordinates we have used to describe the rotating fivebrane covers just one half of the external spacetime. Obviously, the metric can be continued analytically to $\phi \in[0,2 \pi)$; nevertheless, another way to the

\footnotetext{
4 Actually, one might expect the model to represent a limiting, exactly solvable case of a class of solutions to the low-energy action of string theory, quite analogously as in [6]. These lowenergy solutions can be expected to open the throat at infinity. I am indebted to Jeff Harvey for illuminating discussions on this point.
} 
analytic continuation exists. To show this, let us again start with the Lagrangian (1.1), but now gauge

$$
g_{E} \rightarrow h_{E} g_{E} h_{E}^{-1}, \quad g_{M} \rightarrow h_{M}^{\beta} g_{M} h_{M}^{\beta}
$$

Repeating the same story as above, we arrive at

$$
\begin{aligned}
d s_{5 \mathrm{D}}^{2}= & \frac{k_{M}}{4} d r_{M}^{2}+\frac{k_{E}}{4} d r_{E}^{2}+\frac{k_{E} \cosh ^{2} \frac{r_{E}}{2}\left(1+L \cosh ^{2} \frac{r_{M}}{2}\right)}{\sinh ^{2} \frac{r_{E}}{2}+L \cosh ^{2} \frac{r_{M}}{2}} d \theta^{2} \\
& -\frac{k_{M} \sinh ^{2} \frac{r_{M}}{2}\left(L+\sinh ^{2} \frac{r_{E}}{2}\right)}{\sinh ^{2} \frac{r_{E}}{2}+L \cosh ^{2} \frac{r_{M}}{2}} d t^{2}+\frac{2 k_{M} \beta \cosh ^{2} \frac{r_{E}}{2} \sinh ^{2} \frac{r_{M}}{2}}{\sinh ^{2} \frac{r_{E}}{2}+L \cosh ^{2} \frac{r_{M}}{2}} d t d \theta \\
& \quad+\frac{k_{M} \cosh ^{2} \frac{r_{M}}{2} \sinh ^{2} \frac{r_{E}}{2}}{\sinh ^{2} \frac{r_{E}}{2}+L \cosh ^{2} \frac{r_{M}}{2}} d \tilde{t}^{2} \\
B= & \frac{k_{M} \sinh ^{2} \frac{r_{E}}{2} \sinh ^{2} \frac{r_{M}}{2}}{\sinh ^{2} \frac{r_{E}}{2}+L \cosh ^{2} \frac{r_{M}}{2}} d t \wedge d \tilde{t}-\frac{\beta k_{M} \cosh ^{2} \frac{r_{E}}{2} \cosh ^{2} \frac{r_{M}}{2}}{\sinh ^{2} \frac{r_{E}}{2}+L \cosh ^{2} \frac{r_{M}}{2}} d \theta \wedge d \tilde{t} \\
\Phi= & \ln \left(\sinh ^{2} \frac{r_{E}}{2}+L \cosh ^{2} \frac{r_{M}}{2}\right)+\operatorname{const}
\end{aligned}
$$

where we have used the notation of (2.4). Continuing analytically to the $S L(2, \mathbf{R}) \times S U(2)$ gauged model, we obtain (in the notation of (2.7))

$$
\begin{aligned}
d s_{5 \mathrm{D}}^{2}= & \frac{k_{M}}{4} d r_{M}^{2}+\frac{k}{4} d \phi^{2}+\frac{k \cos ^{2} \frac{\phi}{2}\left(1+L \cosh ^{2} \frac{r_{M}}{2}\right)}{\sin ^{2} \frac{\phi}{2}+L \cosh ^{2} \frac{r_{M}}{2}} d \theta^{2} \\
& -\frac{k_{M} \sinh ^{2} \frac{r_{M}}{2}\left(L+\sin ^{2} \frac{\phi}{2}\right)}{\sin ^{2} \frac{\phi}{2}+L \cosh ^{2} \frac{r_{M}}{2}} d t^{2}-\frac{2 k_{M} \beta \cos ^{2} \frac{\phi}{2} \sinh ^{2} \frac{r_{M}}{2}}{\sin ^{2} \frac{\phi}{2}+L \cosh ^{2} \frac{r_{M}}{2}} d t d \theta \\
& +\frac{k_{M} \cosh ^{2} \frac{r_{M}}{2} \sin ^{2} \frac{\phi}{2}}{\sin ^{2} \frac{\phi}{2}+L \cosh ^{2} \frac{r_{M}}{2}} d \tilde{t}^{2} \\
B= & \frac{k_{M} \sin ^{2} \frac{\phi}{2} \sinh ^{2} \frac{r_{M}}{2}}{\sin ^{2} \frac{\phi}{2}+L \cosh ^{2} \frac{r_{M}}{2}} d t \wedge d \tilde{t}+\frac{\beta k_{M} \cos ^{2} \frac{\phi}{2} \cosh ^{2} \frac{r_{M}}{2}}{\sin ^{2} \frac{\phi}{2}+L \cosh ^{2} \frac{r_{M}}{2}} d \theta \wedge d \tilde{t} \\
\Phi= & \ln \left(\sin ^{2} \frac{\phi}{2}+L \cosh ^{2} \frac{r_{M}}{2}\right)+\operatorname{const}
\end{aligned}
$$

which is exactly the analytic continuation of (2.7) from $\phi \in[0, \pi)$ to $\phi \in[\pi, 2 \pi)$.

\section{Exact Axisymmetric Stationary Solutions in Four Dimensions}

Now let us return to our attempt at constructing a 4D exact solution of bosonic string theory by combining two $2 \mathrm{D}$ black holes. The problem we have arrived at is the violation of Witten's non-anomalousness condition (1.14) by the proposed gauge group 
action (1.2). In [5], Witten has shown that even in such anomalous cases, it is possible to choose a gauged Lagrangian in such a way that the gauge non-invariant terms don't depend on the WZW group variable. This leads us to suspect that a $U(1) \times U(1)$ action on $S L(2, \mathbf{R})_{M} \times S L(2, \mathbf{R})_{E}$ exists which is still anomalous on each of the $S L(2, \mathbf{R})$ 's separately, but the gauge non-invariances cancel between sectors $M$ and $E$. This is indeed the case, as we are now going to see.

Let us start with (1.1) once more, and set $k_{M}=k_{E} \equiv k$ for simplicity. The $U(1) \times U(1)$ group to be gauged acts by

$$
\begin{array}{rlrl}
U(1)_{E}: & g_{E} \rightarrow h_{E} g_{E} h_{E}, \quad g_{M} \rightarrow h_{M}^{\alpha} g_{M} h_{M}^{-\alpha}, \\
U(1)_{M}: & g_{E} \rightarrow h_{E}^{\alpha} g_{E} h_{E}^{-\alpha}, & g_{M} \rightarrow h_{M} g_{M} h_{M} .
\end{array}
$$

It is easy to show that (3.1) satisfies condition (1.14). The shift from (1.2) to (3.1) adds new $\alpha$-dependent terms to $(1.10)$,

$$
\begin{aligned}
\mathcal{L}_{4 \mathrm{D}} \rightarrow & \mathcal{L}_{4 \mathrm{D}}-\frac{i k_{M}}{\pi} \int d^{2} z \alpha \sinh ^{2} \frac{r_{M}}{2}\left(A_{E} \bar{\partial} t+\bar{A}_{E} \partial t\right) \\
& -\frac{2 i k_{M}}{\pi} \int d^{2} z \alpha^{2} \sinh ^{2} \frac{r_{M}}{2} A_{E} \bar{A}_{E} \\
+ & \frac{i k_{M}}{\pi} \int d^{2} z \alpha\left(\cosh ^{2} \frac{r_{M}}{2}+\sinh ^{2} \frac{r_{M}}{2}\right)\left(A_{E} \bar{A}_{M}-A_{M} \bar{A}_{E}\right),
\end{aligned}
$$

thus modifying the (lowest order) background fields to

$$
\begin{aligned}
d s_{4 \mathrm{D}}^{2} & =\frac{k}{4} d r_{E}^{2}+\frac{k}{4} d r_{M}^{2}+\frac{k \cosh ^{2} \frac{r_{M}}{2} \sinh ^{2} \frac{r_{E}}{2}}{\Delta} d \theta^{2}-\frac{k \sinh ^{2} \frac{r_{M}}{2} \cosh ^{2} \frac{r_{E}}{2}}{\Delta} d t^{2}, \\
B & =\frac{k \alpha \sinh ^{2} \frac{r_{E}}{2} \sinh ^{2} \frac{r_{M}}{2}}{\Delta} d t \wedge d \theta, \\
\Phi & =\ln (\Delta)+\text { const },
\end{aligned}
$$

where we have shortened $e^{\Phi} \equiv \Delta$, with

$$
\Delta \equiv \cosh ^{2} \frac{r_{M}}{2} \cosh ^{2} \frac{r_{E}}{2}-\alpha^{2} \sinh ^{2} \frac{r_{M}}{2} \sinh ^{2} \frac{r_{E}}{2} .
$$

(3.3) is (the lowest order approximation to) the class of exact, stationary and axisymmetric solutions of four-dimensional string theory advertised above. 0 To avoid naked singularities,

5 This construction can be obviously generalized to $N=1$ superstrings. As the cosets will in fact carry $N=2$ supersymmetry, they will correspond to solutions of $N=1$ superstring theory with $N=1$ supersymmetry in the target. 
we will restrict ourselves to $|\alpha|<1$. (Note the singular behavior of the full Lagrangian (3.2) at the limiting values of $\alpha, \alpha= \pm 1$.)

Now let us analyze shortly the global structure of the solution. The surface at $r_{M}=0$ is an event horizon, which inherits at fixed $t$ the geometry of the Euclidean 2D black hole:

$$
d s_{\text {horizon }}^{2}=\frac{k}{4} d r_{E}^{2}+k \tanh ^{2} \frac{r_{E}}{2} d \theta^{2}
$$

The structure of the horizon suggests that it might be reasonable to interpret the solution as a black string.

The external geometry (3.3) can be continued behind the horizon as follows. As we have remarked above, the Euler angle parametrization we have used does not cover the $S L(2, \mathbf{R})_{M}$ group manifold completely. In another region, the following Euler angles are useful:

$$
g_{M}=\left(\begin{array}{cc}
e^{t_{L} / 2} & 0 \\
0 & e^{-t_{L} / 2}
\end{array}\right)\left(\begin{array}{cc}
\cos \frac{r_{M}}{2} & -\sin \frac{r_{M}}{2} \\
\sin \frac{r_{M}}{2} & \cos \frac{r_{M}}{2}
\end{array}\right)\left(\begin{array}{cc}
e^{-t_{R} / 2} & 0 \\
0 & e^{t_{R} / 2}
\end{array}\right)
$$

with $r_{M} \in(-\pi, \pi)$. In this parametrization of the Lagrangian, we are led to

$$
\begin{aligned}
d s_{4 \mathrm{D}}^{2} & =\frac{k}{4} d r_{E}^{2}-\frac{k}{4} d r_{M}^{2}+\frac{k \cos ^{2} \frac{r_{M}}{2} \sinh ^{2} \frac{r_{E}}{2}}{\Delta_{\text {int }}} d \theta^{2}+\frac{k \sin ^{2} \frac{r_{M}}{2} \cosh ^{2} \frac{r_{E}}{2}}{\Delta_{\text {int }}} d t^{2}, \\
B & =-\frac{k \alpha \sinh ^{2} \frac{r_{E}}{2} \sin ^{2} \frac{r_{M}}{2}}{\Delta_{\text {int }}} d t \wedge d \theta, \\
\Phi & =\ln \left(\Delta_{\text {int }}\right)+\text { const },
\end{aligned}
$$

now with

$$
\Delta_{\text {int }} \equiv \cos ^{2} \frac{r_{M}}{2} \cosh ^{2} \frac{r_{E}}{2}+\alpha^{2} \sin ^{2} \frac{r_{M}}{2} \sinh ^{2} \frac{r_{E}}{2} .
$$

This describes the geometry of the solution behind the horizon. Note that $r_{M}$ has become timelike while $t$ is now spacelike, as might have been expected.

At $r_{M}=\pi$ we encounter a singularity. 1 However, an interesting effect occurs here: While in the direct product geometry of $\alpha=0$ any observer behind the horizon must fall into the singularity (which exists at $r_{M}=\pi$ for any value of $r_{E}$ ), with $\alpha$ nonzero the singularity is localized at $r_{E}=0$. What then happens to the observer at fixed nonzero $r_{E}$ with increasing timelike coordinate $r_{M}$ ? It is easy to see that the internal geometry (3.7)

6 Strictly speaking, this is a future singularity. The same analysis can be carried out for the past singularity at $r_{M}=-\pi$. 
can be continued further to another region. This region corresponds to the remaining part of the $S L(2, \mathbf{R})_{M}$ group manifold, parametrized by

$$
g_{M}=\left(\begin{array}{cc}
e^{t_{L} / 2} & 0 \\
0 & e^{-t_{L} / 2}
\end{array}\right)\left(\begin{array}{cc}
\sinh \frac{r_{M}}{2} & \cosh \frac{r_{M}}{2} \\
-\cosh \frac{r_{M}}{2} & -\sinh \frac{r_{M}}{2}
\end{array}\right)\left(\begin{array}{cc}
e^{-t_{R} / 2} & 0 \\
0 & e^{t_{R} / 2}
\end{array}\right) .
$$

After crossing the event horizon at $r_{M}=0$ in (3.3), the observer can avoid the singularity, cross a new, inner horizon at $r_{M}=\pi$ with $r_{E} \neq 0$, and enter the portion of the universe coming from (3.9). At the inner horizon, $\theta$ becomes timelike, while $r_{M}$ turns spacelike again. The former time coordinate $t$ remains spacelike, and plays the role of an angular variable. The inner horizon carries the geometry of the dual Euclidean 2D black hole:

$$
d s_{\text {horizon }}^{2}=\frac{k}{4} d r_{E}^{2}+\frac{k}{\alpha^{2}} \operatorname{coth}^{2} \frac{r_{E}}{2} d t^{2} .
$$

Hence, the roles of $t$ and $\theta$ have been completely interchanged in the region behind the inner horizon, when compared to the geometry of (3.3). The lowest order background in this region is

$$
\begin{aligned}
d s_{4 \mathrm{D}}^{2} & =\frac{k}{4} d r_{E}^{2}+\frac{k}{4} d r_{M}^{2}-\frac{k \sinh ^{2} \frac{r_{M}}{2} \sinh ^{2} \frac{r_{E}}{2}}{\tilde{\Delta}} d \theta^{2}+\frac{k \cosh ^{2} \frac{r_{M}}{2} \cosh ^{2} \frac{r_{E}}{2}}{\tilde{\Delta}} d t^{2} \\
B & =-\frac{k \alpha \sinh ^{2} \frac{r_{E}}{2} \cosh ^{2} \frac{r_{M}}{2}}{\tilde{\Delta}} d t \wedge d \theta \\
\Phi & =\ln (\tilde{\Delta})+\mathrm{const}, \\
\tilde{\Delta} & \equiv \alpha^{2} \cosh ^{2} \frac{r_{M}}{2} \sinh ^{2} \frac{r_{E}}{2}-\sinh ^{2} \frac{r_{M}}{2} \cosh ^{2} \frac{r_{E}}{2} .
\end{aligned}
$$

This geometry describes, for $r_{M}$ close enough to zero, a throat with a naked singularity. The throat can be continued through its future horizon, and the analysis can be repeated infinitely many times, leading to an infinite strip of geometries and horizons.

Without any computation, the residual (Killing) global symmetry of the coset is (at least) $U(1) \times U(1)$, as precisely these symmetries survive the gauging of (3.1) on $S L(2, \mathbf{R})_{M} \times S L(2, \mathbf{R})_{E}$. It is worth stressing that the $U(1) \times U(1)$ is an exact Killing symmetry of the full-fledged, exact CFT, not just an accidental symmetry of the lowest order background. In this sense, we are guaranteed to have obtained a class of exact, stationary and axisymmetric classical solutions of string theory in four dimensions.

7 Here $r_{M}>0$. The region with $r_{M}<0$ corresponds to the continuation through the analogous horizon in the past, and/or to a 'mirror geometry,' analogously as in, say, the Reissner-Nordstrøm black hole. 
The solution we have just constructed is a twisted product of two $2 \mathrm{D}$ black holes. As the 2D black hole cosets enjoy an interesting property of target duality [12], one might wonder whether there is an analogy of this stringy symmetry for the $4 \mathrm{D}$ cosets. Actually, for general sigma models with a Killing vector, there is a duality transformation [13], given by

$$
\begin{gathered}
\hat{G}_{00}=\frac{1}{G_{00}}, \quad \hat{G}_{0 i}=\frac{B_{0 i}}{G_{00}}, \quad \hat{G}_{i j}=G_{i j}-\left(G_{0 i} G_{0 j}-B_{0 i} B_{0 j}\right) / G_{00} \\
\hat{B}_{0 i}=\frac{G_{0 i}}{G_{00}}, \quad \hat{B}_{i j}=B_{i j}+\left(G_{0 i} B_{0 j}-B_{0 i} G_{0 j}\right) / G_{00} \\
\hat{\Phi}=\Phi+\ln \left(-G_{00}\right)
\end{gathered} .
$$

Note that in the case of our $4 \mathrm{D}$ solution, we obtain $\hat{B}=0$, but instead of nonzero values of the antisymmetric tensor field, nonzero off-diagonal components of the metric tensor occur. When applied to the region represented by (3.3), this duality transformation would give a metric with a naked singularity at $r_{M}=0$, as it is easy to see that (3.12) maps the horizon to a singularity. To obtain a metric without naked singularities, it is a better idea to apply (3.12) to the region behind the naked singularity in (3.11). Indeed, upon doing this we obtain a remarkably simple geometry,

$$
\begin{aligned}
\widehat{d s}_{4 \mathrm{D}}^{2} & =\frac{k}{4} d r_{E}^{2}+\frac{k}{4} d r_{M}^{2} \\
& \quad+k \tanh ^{2} \frac{r_{E}}{2}\left[d \theta^{2}-2 \alpha d t d \theta-\left(\tanh ^{2} \frac{r_{M}}{2} \operatorname{coth}^{2} \frac{r_{E}}{2}-\alpha^{2}\right) d t^{2}\right], \\
\hat{B} & \equiv 0, \\
\hat{\Phi} & =\ln \left(\cosh ^{2} \frac{r_{E}}{2} \cosh ^{2} \frac{r_{M}}{2}\right)+\text { const. }
\end{aligned}
$$

Quite surprisingly, this is a direct product of two 2D black holes! Indeed, upon changing coordinates to $\Theta=\theta-\alpha t, T=t$, we obtain the standard direct product metric of one Euclidean and one Minkowskian black hole, parametrized by $r_{E}, \Theta$ and $r_{M}, T$ respectively. How does it come about that the full class of highly nontrivial spacetimes is dual to a simple, tensor product structure? The crucial point is that (3.12) assumes a preferred Killing vector with respect to which the duality transformation is performed. 8 We have tacitly assumed that this Killing vector coincides with $\partial / \partial t$. Nevetheless, it is a peculiarity

8 Compare the recent discussion of duality by Roček and E. Verlinde in 14]. Note also that there is a similarity between the duality found above, and the twisting procedure studied by Sen in 15$]$. 
of our geometry that there are two commuting Killing vectors in the target, and the duality transformation now requires a choice of preferred basis in the space of Killing vectors. Naively, we can apply (3.12) to any particular choice of the basis, thus obtaining a class of a priori different geometries. The same situation emerges for the tensor product of two black holes, which explains the duality observed above.

We have constructed a class of $U(1) \times U(1)$ symmetric solutions of $4 \mathrm{D}$ string theory, and have found indications of a remarkable duality of the models. In general relativity, $U(1) \times U(1)$ symmetric metrics have attracted much interest, and appealing results have been achieved: not only many physically important exact solutions of Einstein's equations belong to this class, but Einstein's equations become exactly solvable in this limit by inverse scattering methods, in particular multi-monopole solutions can be found, infinite-dimensional solution-generating groups of symmetries exist, explainable via intimate relations to dimensional reduction to $2 \mathrm{D}$, to mention at least some of the crucial aspects of axisymmetric stationary metrics in Einstein gravity. On the other hand, the 2D black hole cosets, which serve as basic building blocks for the constructions of our paper, have been shown recently to enjoy a rich internal structure [16], related in particular to $W_{\infty}$ algebras [17]. It would indeed be desireable to study possible interplays between the deep results of general relativity of axisymmetric stationary geometries on one hand, and string theory with its extremely rich mathematical structure on the other. We hope that the exact solutions we have constructed above might serve as a starting point for further investigation in this direction.

Acknowledgement. It is a pleasure to thank Peter Bowcock, Tohru Eguchi, Jeff Harvey, Elias Kiritsis and Emil Martinec for valuable discussions. 


\section{References}

[1] E. Witten, Phys. Rev. D44 (1991) 314

[2] N. Ishibashi, M. Li and A.R. Steif, 'Two Dimensional Charged Black Holes in String Theory,' Santa Barbara preprint UCSBTH-91-28 (June 1991)

[3] J.H. Horne and G.T. Horowitz, 'Exact Black String Solutions in Three Dimensions,' Santa Barbara preprint UCSBTH-91-39 (July 1991)

[4] S.B. Giddings and A. Strominger, 'Exact Black Fivebranes in Critical Superstring Theory,' Santa Barbara preprint UCSBTH-91-35 (July 1991)

[5] E. Witten, 'On Holomorphic Factorization of WZW and Coset Models,' IAS preprint IASSNS-HEP-91/25 (June 1991)

[6] C.G. Callan, J.A. Harvey and A. Strominger, Nucl. Phys. B359 (1991) 611

[7] D. Garfinkle, G. Horowitz and A. Strominger, Phys. Rev. D43 (1991) 3140

[8] G.W. Gibbons and K. Maeda, Nucl. Phys. B298 (1988) 741

[9] G.T. Horowitz and A. Strominger, Nucl. Phys. B360 (1991) 197

[10] C.G. Callan, Jr., 'Instantons and Solitons in Heterotic String Theory,' Princeton U. preprint PUPT-1278 (June 1991)

[11] R.R. Khuri, Phys. Lett. 259B (1991) 261

[12] A. Giveon, 'Target Space Duality and Stringy Black Holes,' Berkeley preprint LBL30671 (April 1991); R. Dijkgraaf, E. Verlinde and H. Verlinde, 'String Propagation in a Black Hole Geometry,' Princeton preprint IASSNS-HEP-91/22 \& PUPT-1252 (May 1991)

[13] T.H. Buscher, Phys. Lett. 201B (1988) 466

[14] M. Roček and E. Verlinde, 'Duality, Quotients and Currents,' IAS \& Stony Brook preprint IASSNS-HEP-91/68 \& ITP-SB-91-53 (October 1991)

[15] A. Sen, 'Twisted Black $p$-Brane Solutions in String Theory,' Tata preprint TIFR/TH/91-37 (August 1991)

[16] J. Distler and P. Nelson, 'New Discrete States of Strings Near a Black Hole,' PennState \& Princeton preprint UPR-0462T \& PUPT-1262 (August 1991)

[17] I. Bakas and E. Kiritsis, 'Beyond the Large N Limit: Non-linear $W_{\infty}$ as Symmetry of the $\mathrm{SL}(2, \mathrm{R}) / \mathrm{U}(1)$ Coset Model,' Berkeley \& College Park preprint UCB-PTH-91/44 \& LBL-31213 \& UMD-PP-92-37 (September 1991) 